EPJ Web of Conferences 37, 06010 (2012)

DOI: $10.1051 /$ epjconf/20123706010

(C) Owned by the authors, published by EDP Sciences, 2012

\title{
Measurement of the Central Exclusive Production of pion pairs using tagged forward protons at the STAR detector at RHIC
}

\author{
Jacek Turnau ${ }^{\mathrm{a}}$ for the STAR Collaboration \\ Institute for Nuclear Physics PAN, Cracow, Poland
}

\begin{abstract}
We present preliminary measurement of the invariant mass spectrum of the two oppositely charged pions produced in the process $p p \rightarrow p \pi^{+} \pi^{-} p$ (Central Exclusive Production), obtained with the STAR detector at RHIC at $\sqrt{(s)}=200 \mathrm{GeV}$. The Roman Pots were used to tag forward protons and the invariant mass of the pion pair was obtained using tracks reconstructed in the STAR Time Projection Chamber (TPC). The non-exclusive background estimated from events with like-sign track pairs is small, thus proof of principle of this type of the measurement at small momentum transfer to proton is established. Plans for the future are also discussed.
\end{abstract}

\section{Introduction}

Central Exclusive Production (CEP) is a process of the type $A+B \rightarrow A+X+B$ which has the following properties

- Colliding hadrons A and B emerge intact in the final state

- Produced state $\mathrm{X}$ is fully measured

At high energy CEP can be described in terms of the Regge formalism [1] (Fig. 1).

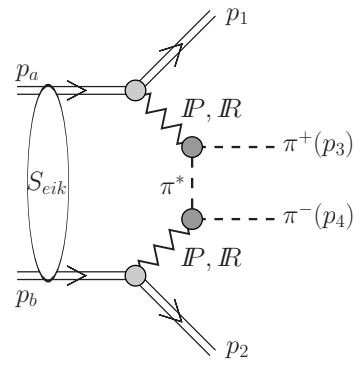

Fig. 1. Central Exclusive Production process interpreted in terms of the Regge trajectory exchange At collider energy Pomeron $\mathcal{P}$ dominates other lower lying Regge trajectories. $\mathcal{R}$

Of special interest is a particular case when $\mathrm{A}, \mathrm{B}$ and $\mathrm{X}$ are separated by large rapidity gaps (e.g. > 3 units of rapidity). Then, Pomeron dominates other Regge trajectories by large factor, and for purely hadronic processes CEP is almost equivalent to Double Pomeron Exchange (DPE). DPE is a spin parity filter i.e. system X produced in DPE has even spin and positive parity. It should be mentioned that in CEP vector particles can also be produced in diffractive photoproduction process. Although much weeker than DPE, it is still separable due to DPE spin-parity filter property.

\footnotetext{
a e-mail: Jacek.Turnau@ifj.edu.pl
}

This is an Open Access article distributed under the terms of the Creative Commons Attribution License 2.0, which permits unrestricted use, distribution, and reproduction in any medium, provided the original work is properly cited. 
Invariant mass of centrally produced system X depends on the collider energy. At the LHC it can reach above $100 \mathrm{GeV}$ so for example central production of Higgs boson and supersymmetric particles is possible. At TEVATRON it can reach masses up to $100 \mathrm{GeV}$, studies of heavy meson production and pQCD in general are possible (see e.g. [2] for a recent review) At ISR and RHIC we are in the low mass region, so, our physics motivation is spectroscopy, glueball search and non-perturbative aspects of meson production. For understanding of the scalar meson spectrum CEP has advantage of the above mentioned isospin-spin-parity filter which simplifies partial wave analysis. In addition there is general agreement that lightest glueball should be a scalar meson with mass in range 1-2 GeV. Due to high gluon density in the Pomeron, glueball formation might be enhanced in DPE. Another topic of interest is a non-perturbative aspect of meson production $[3,5,4]$.

\section{Detector description and selection of the data}

The figure 2 shows schematic view of the detector setup. For tagging forward, di ffractively scattered

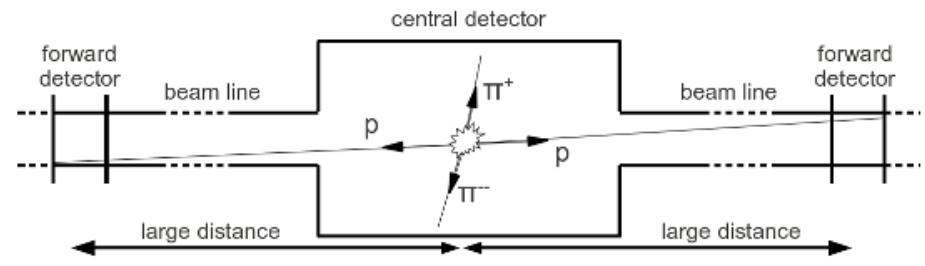

Fig. 2. Schematic view of the detector setup for studies of Central Exclusive Production processes.

protons, Roman Pots with silicon strip detectors positioned at 55.5 and 58.5 meters form Interaction Point (IP) have been used (see [6] for more details). The momentum transfer to tagged protons is in the range .003 to $.03 \mathrm{GeV}^{2}$. For the measurement of centrally produced mesons we use TPC of STAR detector at RHIC [7], high resolution tracking device, covering 2 units of rapidity in the central region. It has $\mathrm{dE} / \mathrm{dx}$ particle identification capability.

We study polarized pp collisions at $\sqrt{(}(s)=200 \mathrm{GeV}$, STAR run in 2009. Data sample is selected using the following requirements:

- Roman Pot trigger i.e. scintillator counter signal consistent with one particle on each side of the IP,

- one reconstructed proton, using Si detectors in RP on each side of IP,

- protons are not collinear (space angle between directions of protons $\Delta \Theta>0.15 \mathrm{mrad}$ ),

- two and only two opposite charge tracks in TPC, coming from the primary vertex $(D C A<2 \mathrm{~cm})$, with transverse momentum $p_{T}>150 \mathrm{MeV}$, pseudorapidity $|\eta|<1$ and $>14$ TPC hits/track,

- transverse momentum balance between produced mesons and forward protons $p_{T}^{\text {miss }}<0.02 \mathrm{GeV}$,

- both tracks are identified as pions $\left(d E / d x-<d E / d x>_{\pi} \mid<3 \sigma\right)$.

For the requirement of transverse momentum balance it is assumed that proton momentum is 100 $\mathrm{GeV}$. We do not measure proton momentum, but we know that in our sample proton momentum loss is few percent, so for the transverse momentum balance it can be safely neglected. The selected sample contains 588 events.

\section{Results}

In figure 3 the spectrum of the invariant mass of $\pi^{+} \pi^{-}$pairs produced in central exclusive process is presented. There is very small like-sign background, which gives a measure of exclusivity of the 
process. The spectrum is not corrected for acceptance, but preliminary acceptance study indicates that corrections will not change shape of the spectrum significantly. Presented spectrum is similar to the one published by AFS Collaboration at ISR [8] i.e.it shows the same characteristic features :

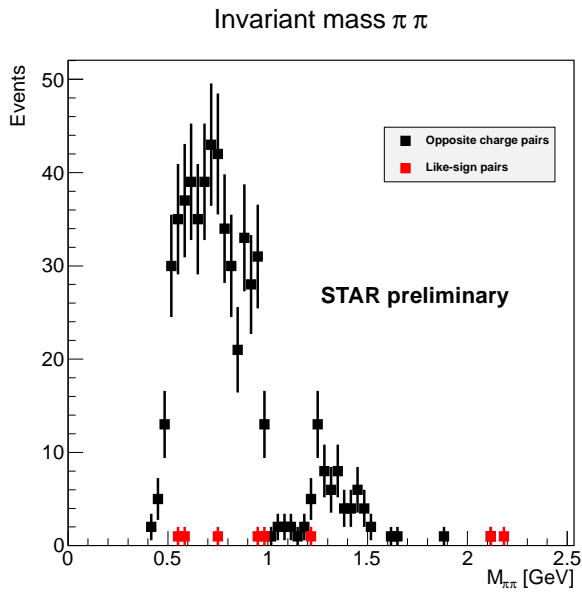

Fig. 3. Invariant mass spectrum of $\pi^{+} \pi^{-}$pairs produced in Central Exclusive process $p+p \rightarrow p+\pi^{+} \pi^{-}+p$ at $200 \mathrm{GeV}$.

- it is dominated by low invariant mass pairs, $m_{\pi \pi}$ below $1 \mathrm{GeV}$,

- shows the same characteristic drop around $1 \mathrm{GeV}$ which is due to $f_{0}(980)$ in the final state interaction [9].

\section{Summary and outlook}

In summary, it has been demonstrated that STAR detector with forward proton taggers has potential to measure exclusive production of mesons with small non-exclusive background estimated by likesign events content of the sample. Further studies of CEP at $200 \mathrm{GeV}$ are in progress. Preparation for analogous measurement at $500 \mathrm{GeV}$ next year is also in progress. Phase II of CEP measurment with standard beam optics is considered. It would allow for very large statistics and higher momentum transfers.

\section{References}

1. A. C. Irving and R. P. Worden, Phys. Rept. 34, 117 (1977).

2. M. G. Albrow, T. D. Coughlin and J. R. Forshaw, Prog. Part. Nucl. Phys. 65 (2010) 149.

3. L. A. Harland-Lang, V. A. Khoze, M. G. Ryskin and W. J. Stirling, arXiv:1204.4803 [hep-ph].

4. R. Staszewski, P. Lebiedowicz, M. Trzebinski, J. Chwastowski and A. Szczurek, Acta Phys. Polon. B 42, (2011) 1861.

5. P. Lebiedowicz and A. Szczurek, Phys. Rev. D 81, (2010) 036003.

6. L. Adamczyk et al. [STAR Collaboration], arXiv:1206.1928 [nucl-ex].

7. K. H. Ackermann et al. [STAR Collaboration], Nucl. Instrum. Meth. A 499, (2003) 624.

8. T. Akesson et al. [Axial Field Spectrometer Collaboration], Nucl. Phys. B 264, (1986) 154.

9. J. -P. Dedonder, A. Furman, R. Kaminski, L. Lesniak and B. Loiseau, Acta Phys. Polon. B 42, (2011) 2013. 